\title{
Glucose ceramide synthase inhibitors prevent osteoclast activation and limit myeloma-induced osteolytic lesions
}

PP160

\author{
Adel Ersek ${ }^{1}, \mathrm{Ke} \mathrm{Xu}^{2}{ }^{2}$ Anastasios Karadimitris ${ }^{2}$, Nicole J. Horwood ${ }^{1}$
}

1 The Kennedy Institute of Rheumatology, University of Oxford, ${ }^{2}$ Department of Haematology, Hammersmith

Hospital, Imperial College London

\section{INTRODUCTION}

Glycosphingolipids (GSL) are essential structural components of mammalian cell membranes and lipid rafts. Cancer associated GSL promote tumour growth, angiogenesis and metastasis; however their role in osteoclast (OC) activation and the development of osteolytic bone diseases such as multiple myeloma (MM) are not known.

In the present study we have investigated the hypothesis that GSL contribute to OC activation and inhibitors of GSL biosynthesis would antagonise GSL-dependent osteoclastogenesis.

\section{METHODS}

8 weeks old C57BL/6 female mice were treated with PBS, GM3 $(10 \mu \mathrm{g})$ and/or $5000 \mathrm{mg} / \mathrm{kg} /$ day N-butyl-deoxynojirimycin (NB-DNJ) for 6 days and bone marrow (BM) cells induced to form OC. The 5TGM1 mouse myeloma model was established by transplanting $10^{\wedge} 6$ 5TGM1-GFP cells to 10 weeks old C57BL/KaLwRij mice. Animals were treated with PBS or 2500 $\mathrm{mg} / \mathrm{kg} / \mathrm{day}$ NB-DNJ for 21 days and BM tumour burden and bone parameters were evaluated by micro-computed tomography.

RESULTS: The GSL GM3 promotes human OCL formation; while GSL inhibitors such as NB-DNJ prevent osteoclastogenesis in vitro.

By using human monocytes that were induced to form OC we have found that osteoclastogenesis was stimulated in a dose dependent manner by GM3. Inhibition of GSL synthesis by NB-DNJ reduced human monocytes osteoclastogenesis capacity in a dose dependent manner.

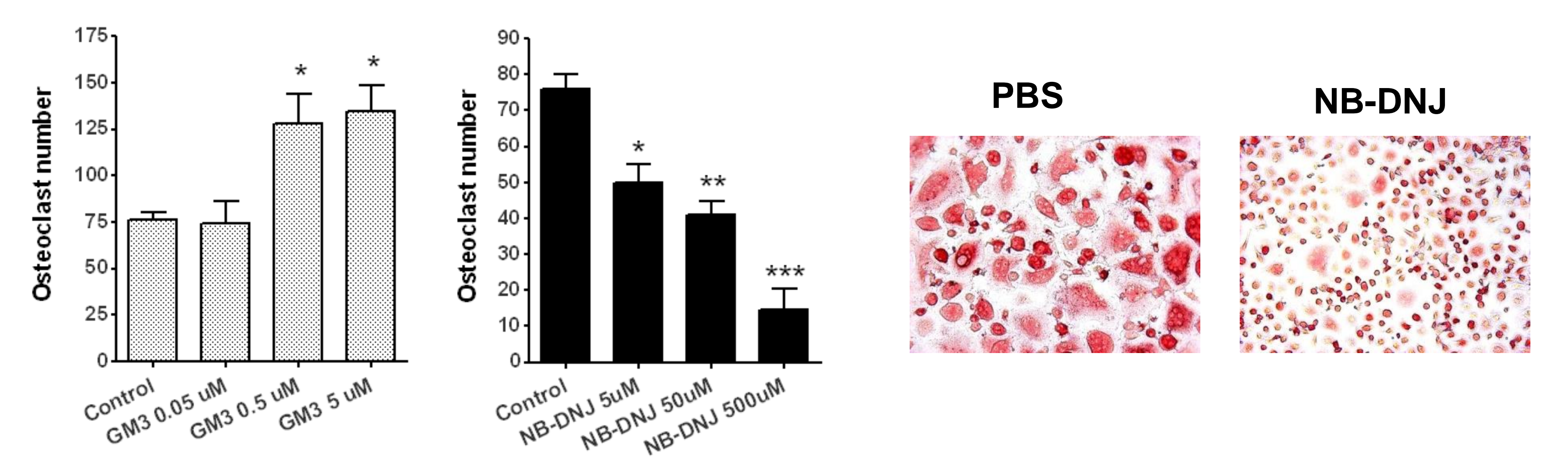

Figure 1: Enumeration of multinucleated $O C$ after tartrate resistant acid phosphatase (TRAP) staining. Cells were cultured in the presence of $25 \mathrm{ng} / \mathrm{ml} \mathrm{M-CSF}$ and $5 \mathrm{ng} / \mathrm{ml}$ RANKL for 7 days $\left({ }^{*} \mathrm{p}<0.05,{ }^{* *} \mathrm{p}<0.01\right.$, ${ }^{* * \star}$ $\mathrm{p}<0.001$ versus control; Original magnification 20X)

RESULTS: GM3 co-operates with RANKL and promotes MAPK phosphorylation during osteoclasogenesis.

The addition of GM3 to developing OCs led to a rapid phosphorylation of ERK, p38 and, to a lesser extent, JNK. Inhibition of GSL biosynthesis by NB-DNJ partially prevented GM3 induced p38, ERK and somewhat JNK phosphorylation.

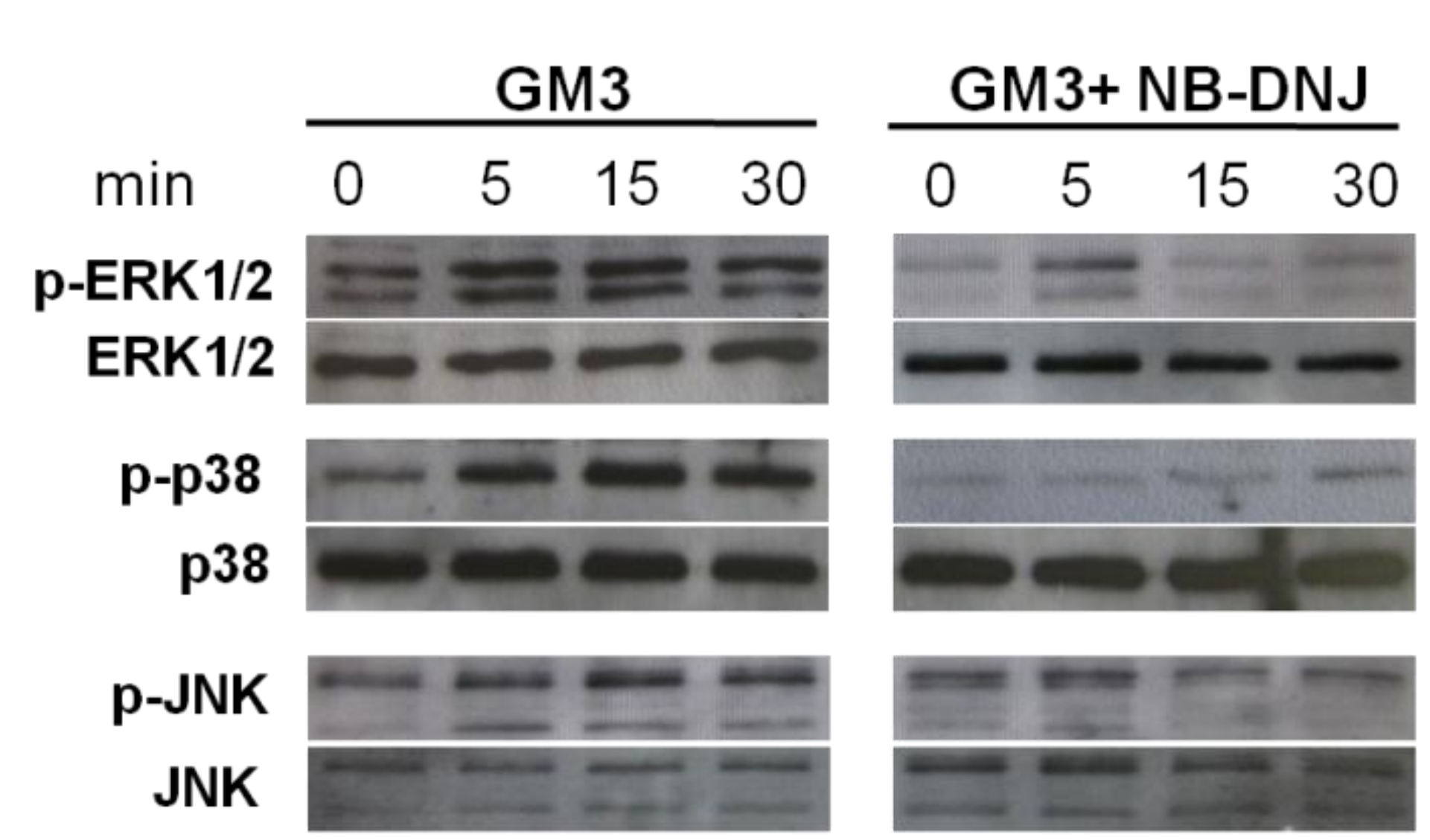

Figure 2: GSL inhibitors block MAPK phosphorylation during osteoclastogenesis. Mouse BM cells were cultured with M-CSF+RANKL

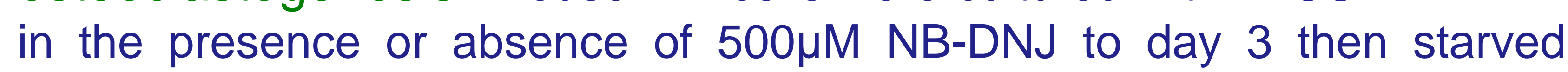
overnight followed by treated with GM3 or GM3+NB-DNJ for the time indicated and immunoblotted with $\alpha$-pERK1/2, a-pp38, a-pJNK Ab. Membranes were stripped and reblotted with $\alpha$-ERK, $\alpha-p 38, \alpha-J N K$ Ab.
RESULTS: GSL synthesis inhibitor NB-DNJ inhibits GM3 proosteoclastogenic effect ex-vivo.

Ex-vivo OC assay set up with BM cells obtained from NB-DNJ treated mice showed reduced numbers of OCL. However, osteoclastogenesis was accentuated in the case of GM3 treated mice. NB-DNJ treatment prevented GM3 induced OCL activation as shown by the TRAP assay but also by the analysis of in situ OC development on histological bone sections.
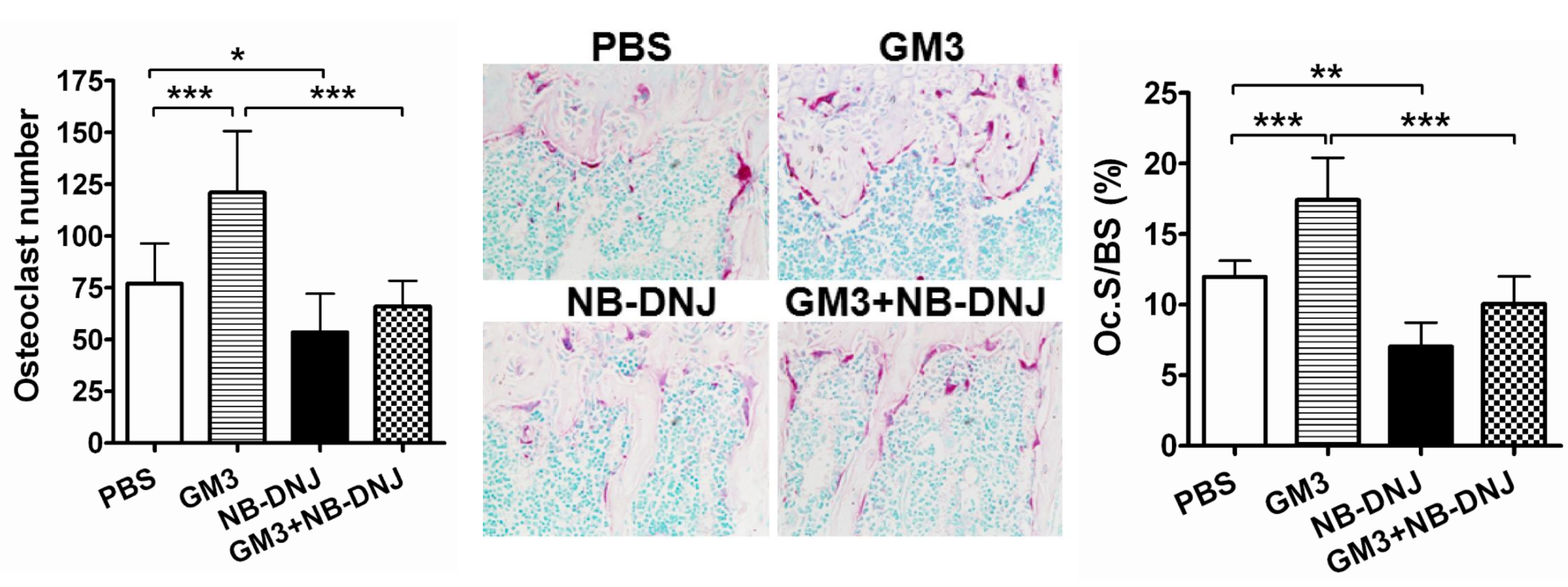

Figure 3: NB-DNJ inhibits GM3-mediated enhanced osteoclast development and activity in vivo. OC TRAP assay from mice treated with GM3 and/or NB-DNJ after 7 days of culture. Histological sections of mouse tibiae stained for OC TRAP activity $\left({ }^{*} p<0.05,{ }^{* \star} p<0.01,{ }^{* \star *} p<0.001\right.$; magnification 20X).

RESULTS: NB-DNJ effectively reduces osteolytic bone lesions in 5TGM1 murine myeloma model.

Inhibition of GSL synthesis with NB-DNJ treatment was able to decrease bone loss in vivo and reduce myeloma-induced lytic bone lesions as shown by a significant increase in bone parameters.
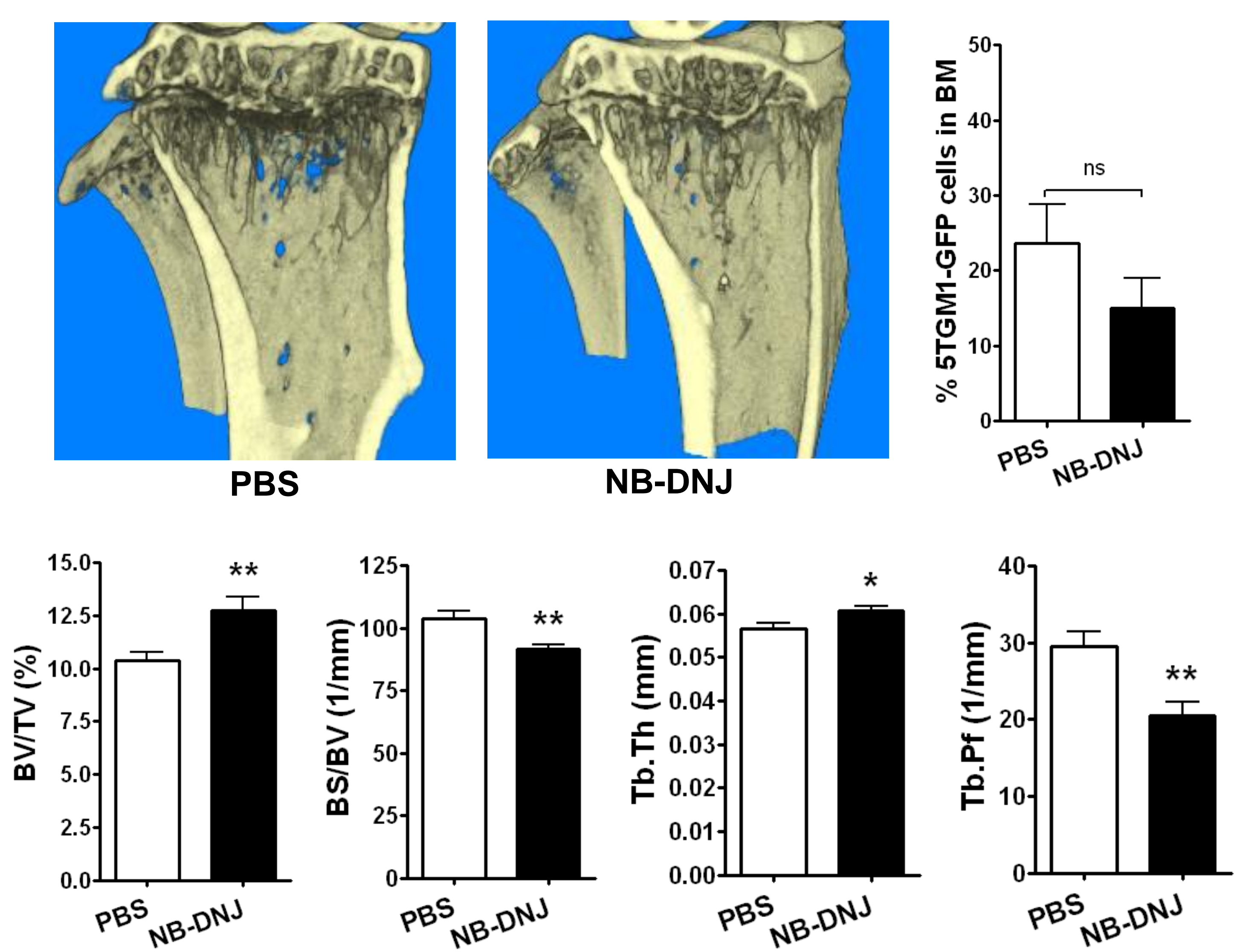

Figure 4: NB-DNJ effectively inhibits 5TGM1 myeloma cells caused bone lesions in C57BL/KaLwRijHsd mice. Longitudinal cross-section of diseased and PBS or NB-DNJ treated mouse tibiae and bone indices evaluated after micro-CT reconstruction.

\section{CONCLUSIONS}

A novel role of OCL activating factor was identified for tumour-derived, as well as of de novo-synthesised GSL GM3. GM3 stimulated osteoclastogenesis by enhancing RANKL ability to induce OCL maturation via increased MAPK phosphorylation.

Inhibiting glycosphingolipid biosynthesis with NB-DNJ effectively reduced baseline as well as GM3-induced OC development and function in vitro and in vivo.

NB-DNJ limited multiple myeloma induced osteolytic lesions and cortical bone perforation improving significantly the trabecular bone architecture when compared to control mice. 